International Journal of Fuzzy Logic Systems(IJFLS) Vol.7, No.3, October 2017

\title{
RESEARCH ON WIND ENERGY INVESTMENT DECISION MAKING: A CASE STUDY IN JILIN
}

\author{
Wei Dong, Cunbin Li, Jiahang Yuan \\ (School of Economics and Management, North China Electric Power University, Beijing, \\ China 102206)
}

\begin{abstract}
:
As a clean renewable energy, wind energy has been focused on an alternative source of fossil fuels. Evaluating wind energy investment projects including multiple attributes is a multi-attribute decision making (MADM) problem. Due to the strong ability in expressing the fuzziness and uncertainty, this paper uses intuitionistic trapezoidal fuzzy numbers to describe the evaluation information from decision-makers. Considering the interaction among attributes and the influence of subjective risk, this paper proposes an intuitionistic trapezoidal fuzzy aggregation operator based on Choquet integral and prospect theory. We use the new operator to aggregate the prospect value functions. And then we generate a grey relationprojection pursuit dynamic cluster method to rank the alternatives. At last, an illustrative example has been taken to demonstrate the validity and feasibility of the proposed method.
\end{abstract}

\section{Key words:}

Wind energy; Decision making; Intuitionistic trapezoidal fuzzy number; Choquet integral

\section{INTRODUCTION}

Among renewable energy resources, wind energy is one of themost profitable, clear and powerful way to supply the applicationsof renewable energy[1], during the time that the generation of thewind energy has increased $32 \%$ per year in the past 10 years[2]. Wind energy is more environmentallyfriendly and does not cause pollution. Since wind energy isproduced from natural, recurring and continuous outflow of energy[3], and does not consume any natural resource[4] and can benaturally replenished [5], it is also sustainable.This paper aims to study on wind energy investment decision making for Jilin province of China.Jilin is in the high latitude area, so its wind energy reserves are relatively abundant. Nationwide, the total amount of wind energy resources in Jilin ranks fifth in the country. According to the reserves and availability of wind energy, Jilin can be divided into two areas, namely, abundant area and available area.

Selecting the best solution of wind investment project among alternatives under some attributes is a multi-attribute decision making(MADM) problem. Many scholars has studied on the MADM for renewable energy planning. Sagbansua and Balo (2017) proposes a novel MADM method for selecting a convenient turbine from various perspectives for developing a wind energy station. The method combines AHP with TOPSIS[6]. Although the process is easy to realize, the decision making contains too much subjective information. Laska (2017) presents a discussion on different methods of multi-criteria analysis and different rules of proceedings that have to be taken into account when making a decision regarding the location of a wind farm in the NE Poland. In the study, six multi-criteria analyses were discussed taking into account the main criteria on which they are based: utility functions (MAUT, AHP, DEMATEL), relationships outranking (ELECTRE, PROMETHEE), and decision support (Borda ranking methods)[7].Villacreses 
et.al(2017) uses MADM method to select the most feasible location for installing wind power plants in continental Ecuador. In addition, a standardization process was performed, which consists of establishing an overall performance index to evaluate the results[8].Elzarka et.al (2017) proposes a vague set fuzzy multi-attribute group decision-making model to select the most appropriate renewable energy technology for institutional owners. The proposed model integrates group rational behavior theory with vague set fuzzy theory. The group rational behavior theory is utilized to account for the varying level of expertise and opinions of decision makers. The vague set fuzzy theory is utilized because most of the collected opinions from decision makers involve fuzzy data and information[9].

For the vagueness of evaluation information, intuitionistic trapezoidal fuzzy numbers are suitable for expressing the fuzziness and uncertainty. In consideration of risk attitude of decision makers, we propose an intuitionistic trapezoidal fuzzy aggregation operator based on Choquet integral and prospect theory. Facing at the MADM problem of wind energy, a grey relation - projection pursuit dynamic cluster method based on intuitionistic trapezoidal fuzzy Choquet integral prospect operator is presented.The fuzzy measures of decision makers are given from subjective preference. The intuitionistic trapezoidal fuzzy numbers are aggregated by the proposed operator, and then the alternatives ranking is obtained by grey relation with projection pursuit dynamic cluster method. From the ranking result, we get the best alternative.

\section{INTUITIONISTIC TraPEZOIDAL FUZZY NUMBER}

Definition 1 [10]. Let $A=\left\langle\left([a, b, c, d] ; \mu_{A}\right),\left(a c^{\prime} b c d ' d^{\prime} \xi_{A}\right\rangle\right)$ be an intuitionistic trapezoidal fuzzy number in the universe of discourse $X$, whose membership function and non-membership function are defined as follows:

$$
\mu_{\bar{A}}(x)=\left\{\begin{array}{ll}
\frac{x-a}{b-a} \mu_{\bar{A}}, & \text { if } a \leq x<b \\
\mu_{\bar{A}}, & \text { if } b \leq x \leq c \\
\frac{d-x}{d-c} \mu_{\bar{n}}, & \text { if } c<x \leq d \\
0, & \text { if } x<\text { a or } x>d
\end{array} \text {, nnd } \quad v_{\bar{A}}(x)= \begin{cases}\frac{b-x+\left(x-a^{\prime}\right) v_{\bar{A}}}{b-a^{\prime}}, & \text { if } a^{\prime} \leq x<b \\
\frac{v_{\bar{A}},}{x-c+\left(d^{\prime}-x\right) v_{\bar{A}}} & \text { if } b \leq x \leq c \\
\frac{d^{\prime}-c}{1,} & \text { if } x<x \leq d^{\prime} \\
1, & \text { if } x>d^{\prime}\end{cases}\right.
$$

respectively, where $a, b, c, d, a^{\prime}, d^{\prime}$ are real numbers, $0 \leq \mu_{A} \leq 1, \quad 0 \leq v_{A} \leq 1,0 \leq \mu_{\AA}+v_{A} \leq 1$. If $a=a^{\prime}, d=d^{\prime}$, the intuitionistic trapezoidal fuzzy number is simplified to $A=\left([a, b, c, d] ; \mu_{\AA}, v_{A}\right)$ which is the study content of this paper.

Definition 2.Let $\AA_{1}=\left(\left[a_{1}, b_{1}, c_{1}, d_{1}\right] ; \mu_{A_{1}}, v_{\bar{A}_{1}}\right)$ and $\AA_{2}=\left(\left[a_{2}, b_{2}, c_{2}, d_{2}\right] ; \mu_{\bar{A}_{2}}, v_{\bar{A}_{2}}\right)$ be two intuitionistic trapezoidal fuzzy numbers in the universe of discourse $X$. The Hamming distance between $A_{1}$ and $A_{2}$ is: 
International Journal of Fuzzy Logic Systems (IJFLS) Vol.7, No.2, October 2017

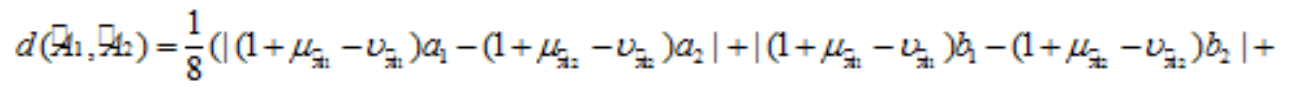

$$
\begin{aligned}
& \left.\left|\left(1+\mu_{\hbar_{1}}-v_{\hbar_{1}}\right) c_{1}-\left(1+\mu_{\hbar_{1}}-v_{\bar{A}_{2}}\right) c_{2}\right|+\left|\left(1+\mu_{\hbar_{1}}-v_{\hbar_{1}}\right) d_{1}-\left(1+\mu_{\hbar_{1}}-v_{\bar{A}_{2}}\right) d_{2}\right|\right)
\end{aligned}
$$

Definition 3. Let $A_{1}=\left(\left[a_{1}, b_{1}, c_{1}, d_{1}\right] ; \mu_{A_{1}}, v_{A_{1}}\right)$ and $A_{2}=\left(\left[a_{2}, b_{2}, c_{2}, d_{2}\right] ; \mu_{A_{2}}, v_{A_{2}}\right)$ be two intuitionistic trapezoidal fuzzy numbers in the universe of discourse $X . T_{\bar{A}_{1}}=\left[a_{1}, b_{1}, c_{1}, d_{1}\right], T_{\bar{A}_{2}}=\left[a_{2}, b_{2}, c_{2}, d_{2}\right]$ are traditional trapezoidal fuzzy numbers, $\left\|T_{\lambda_{1}}^{\lambda}\right\|=\left|\frac{(1-\lambda)\left(a_{1}+b_{1}\right)+\lambda\left(c_{1}+d_{1}\right)}{4}\right|$,

$\left\|T_{x_{2}}^{\lambda}\right\|=\left|\frac{(1-\lambda)\left(a_{2}+b_{2}\right)+\lambda\left(c_{2}+d_{2}\right)}{4}\right|$. The algorithms of intuitionistic trapezoidal fuzzy numbers are:

(1) Addition. $A_{1} \oplus A_{2}=\left(\left[a_{1}+a_{2}, b_{1}+b_{2}, c_{1}+c_{2}, d_{1}+d_{2}\right] ; \frac{\left\|T_{\AA_{1}}^{\lambda}\right\| \mu_{A_{1}}+\left\|T_{\AA_{2}}^{\lambda}\right\| \mu_{\AA_{2}}}{\left\|T_{\lambda_{1}}^{\lambda}\right\|+\left\|T_{\lambda_{2}}^{\lambda}\right\|}, \frac{\left\|T_{\AA_{1}}^{\lambda}\right\| v_{\AA_{2}}+\left\|T_{\lambda_{2}}^{\lambda}\right\| v_{\bar{A}_{2}}}{\left\|T_{\lambda_{1}}^{\lambda}\right\|+\left\|T_{\lambda_{2}}^{\lambda}\right\|}\right)$

(2) Subtraction. $A_{1}$ ! $A_{2}=\left(\left[a_{1}-d_{2}, b_{1}-c_{2}, c_{1}-b_{2}, d_{1}-a_{2}\right] ; \frac{\left\|T_{\lambda_{1}}^{\lambda}\right\| \mu_{\AA_{1}}+\left\|T_{\lambda_{2}}^{\lambda}\right\| \mu_{\AA_{2}}}{\left\|T_{\AA_{1}}^{\lambda}\right\|+\left\|T_{\AA_{2}}^{\lambda}\right\|}, \frac{\left\|T_{\lambda_{1}}^{\lambda}\right\| v_{\bar{A}_{2}}+\left\|T_{\lambda_{2}}^{\lambda}\right\| v_{\bar{A}_{2}}}{\left\|T_{\bar{\lambda}_{1}}^{\lambda}\right\|+\left\|T_{\AA_{2}}^{\lambda}\right\|}\right)$

From the algorithms, we find that $\mu_{\AA_{1} \oplus \AA_{2}}=\mu_{\AA_{1} ! \AA_{2}}=\frac{\left\|T_{\bar{\lambda}_{1}}^{\lambda}\right\| \mu_{\AA_{1}}+\left\|T_{\lambda_{2}}^{\lambda}\right\| \mu_{\AA_{2}}}{\left\|T_{\AA_{1}}^{\lambda}\right\|+\left\|T_{\AA_{2}}^{\lambda}\right\|}$,

$$
v_{\bar{A}_{1} \oplus \AA_{2}}=v_{\bar{A}_{1} ! \AA_{2}}=\frac{\left\|T_{\AA_{1}}^{\lambda}\right\| v_{\bar{A}_{1}}+\left\|T_{\lambda_{2}}^{\lambda}\right\| v_{\bar{A}_{2}}}{\left\|T_{\AA_{1}}^{\lambda}\right\|+\left\|T_{\AA_{2}}^{\lambda}\right\|} .
$$

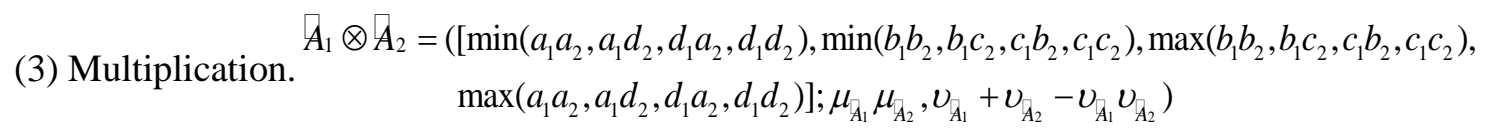

(4) Scalar-multiplication. $\chi \AA_{1}=\left\{\begin{array}{l}\left(\left[\chi a_{1}, \chi b_{1}, \chi c_{1}, \chi d_{1}\right] ; \mu_{\AA_{1}}, v_{\bar{A}_{1}}\right), \chi \geq 0 \\ \left(\left[\chi d_{1}, \chi c_{1}, \chi b_{1}, \chi a_{1}\right] ; \mu_{\overparen{A}_{1}}, v_{\AA_{1}}\right), \chi<0\end{array}\right.$ 
(5) Exponentiation

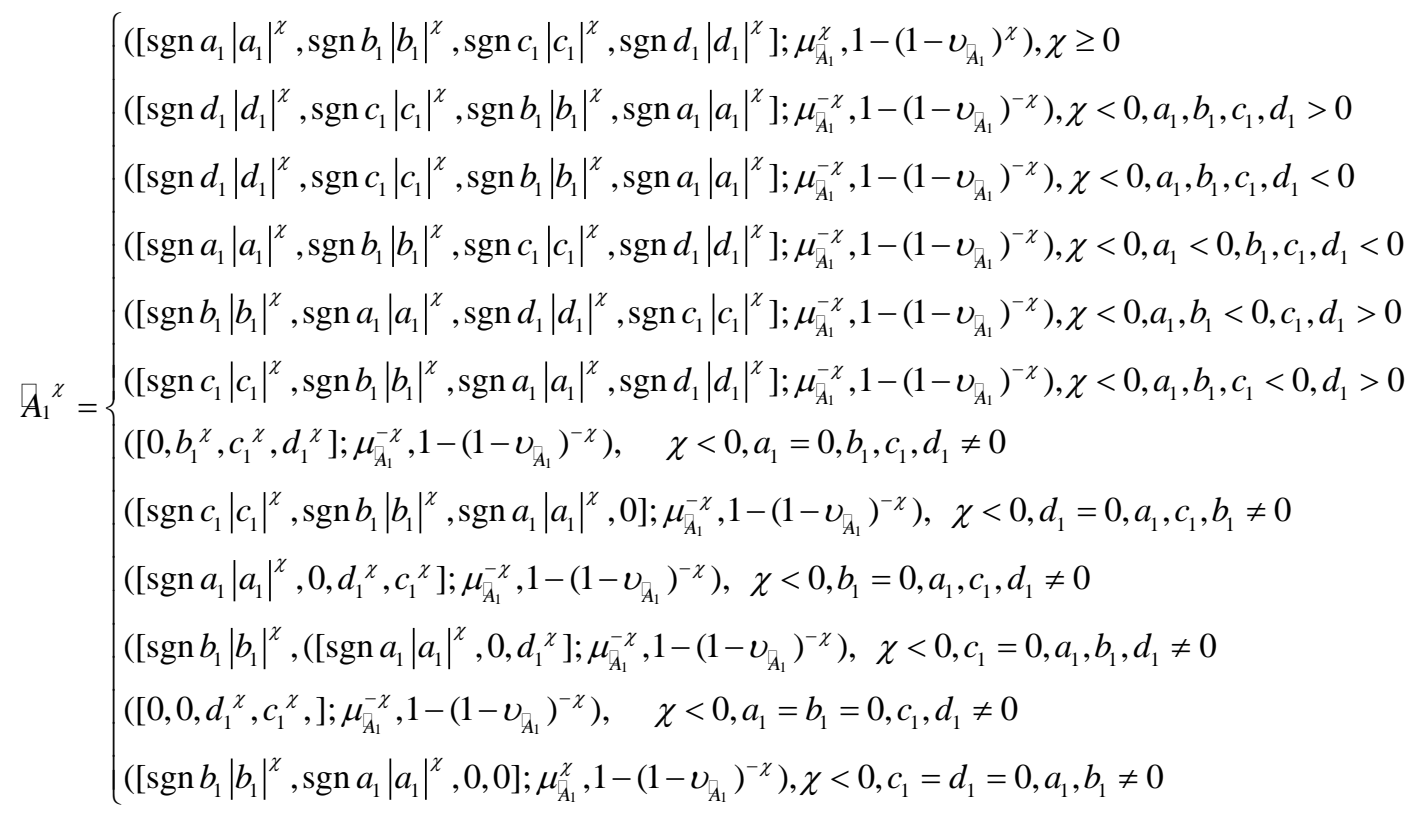

Where sgn is a sign function, $\operatorname{sgn} x=\left\{\begin{array}{ll}1, & x>0 \\ 0, & x=0 \\ -1, & x<0\end{array} \quad x \in R\right.$

(6) Division. If $a_{2}, b_{2}, c_{2}, d_{2} \neq 0$, then

$$
\begin{aligned}
\AA_{1} / A_{2}= & \left(\left[\min \left(a_{1} / a_{2}, a_{1} / d_{2}, d_{1} / a_{2}, d_{1} / d_{2}\right), \min \left(b_{1} / b_{2}, b_{1} / c_{2}, c_{1} / b_{2}, c_{1} / c_{2}\right), \max \left(b_{1} / b_{2}, b_{1} / c_{2}, c_{1} / b_{2}, c_{1} / c_{2}\right),\right.\right. \\
& \left.\left.\max \left(a_{1} / a_{2}, a_{1} / d_{2}, d_{1} / a_{2}, d_{1} / d_{2}\right)\right] ; \mu_{\bar{A}_{1}} \mu_{\bar{A}_{2}}, v_{\bar{A}_{1}}+v_{\bar{A}_{2}}-v_{\bar{A}_{1}} v_{\bar{A}_{2}}\right)
\end{aligned}
$$

\section{Aggregation Operators}

\subsection{Intuitionistic trapezoidal fuzzy Choquet integral (ITFCI)}

Definition 4[11]. Suppose that $\mu$ is the fuzzy measure in the universe of discourse $X=\left\{x_{1}, x_{2}, \ldots, x_{n}\right\}$ and $A_{i}=\left(\left[a_{i}, b_{i}, c_{i}, d_{i}\right] ; \mu_{i}, v_{i}\right)(i=1,2, \ldots, n)$ are intuitionistic trapezoidal fuzzy numbers based on $X$. Then the discrete Choquet integral of fuzzy measure $\mu$ is defined as follows: 


$$
\begin{aligned}
& \int \AA d u=\operatorname{ITFCI}\left(\AA_{1}, \AA_{2}, \ldots, \AA_{n}\right)=\AA_{\tau(1)}\left[\mu\left(B_{\tau(1)}\right)-\mu\left(B_{\tau(2)}\right)\right] \oplus \ldots \oplus \AA_{\tau(n)}\left[\mu\left(B_{\tau(n)}\right)-\mu\left(B_{\tau(n+1)}\right)\right] \\
& =\sum_{i=1}^{n} \AA_{\tau(i)}\left[\mu\left(B_{\tau(i)}\right)-\mu\left(B_{\tau(i+1)}\right)\right]
\end{aligned}
$$

Where $\left(\AA_{\tau(1)}, \AA_{\tau(2)}, \ldots, \AA_{\tau(n)}\right)$ is the permutation of $\left(\AA_{1}, \AA_{2}, \ldots, A_{n}\right)$, for any $i, \AA_{\tau(i)} \leq \AA_{\tau(i+1)}$;

$$
B_{\tau(i)}=\left(x_{\tau(i)}, x_{\tau(i+1)}, \ldots, x_{\tau(n)}\right), B_{\tau(n+1)}=0 \text {. }
$$

\subsection{Intuitionistic trapezoidal fuzzy Choquet integral prospect operator}

In order to solve the problem of subjective risk attitude, prospect theory is proposed in 1979. It shows that decision makers produce different attitude with benefit or cost, which means the expected theory is wrong in the risk decision making with psychological factors. Based on the prospect theory, we put forward the intuitionistic trapezoidal fuzzy Choquet integral prospect (ITFCIP) operator

Definition 5 [12]. Let $\AA_{i}=\left(\left[a_{i}, b_{i}, c_{i}, d_{i}\right] ; \mu_{i}, v_{i}\right)(i=1,2, \ldots, n)$ be a set of intuitionistic trapezoidal fuzzy numbers. Let $R=([a, b, c, d] ; \mu, v)$ be the reference point based on prospect theory, where $\mu$ is the expected membership function and $v$ is the expected non-membership function. Then the deviation degree between $A_{i}$ and $R$ is:

$$
\Delta \AA_{i}=\AA_{i} ! R=\left(\left[a_{i}-d, b_{i}-c, c_{i}-b, d_{i}-a\right] ; \mu_{A ! R}, v_{A ! R}\right)
$$

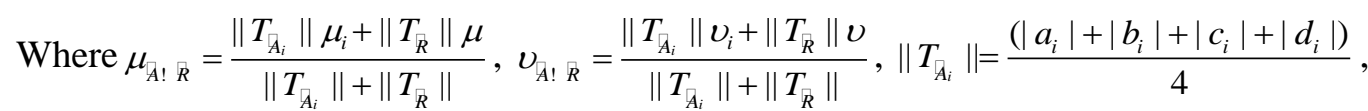

$\left\|T_{R}\right\|=\frac{(|a|+|b|+|c|+|d|)}{4}$.

Definition 6 [12]. Let $\Delta A_{i}=\left(\left[a_{i}-d, b_{i}-c, c_{i}-b, d_{i}-a\right] ; \mu_{A ! R}, v_{A ! R}\right)$ be a set of intuitionistic trapezoidal fuzzy deviation degree, then the intuitionistic trapezoidal fuzzy prospect value function of $\AA_{i}$ is:

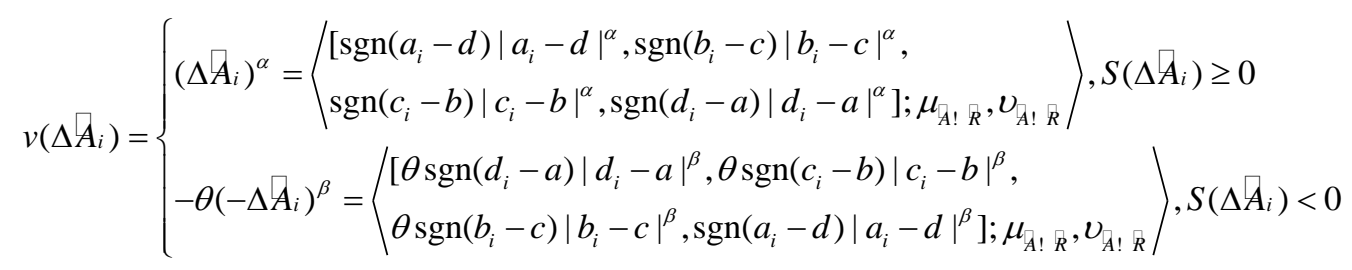

Where $\Delta \AA_{i}$ is the deviation degree between $\AA_{i}$ and $R, S\left(\Delta \rrbracket_{i}\right)$ is the score function of $\Delta \AA_{i}$, $S\left(\Delta \AA_{i}\right)=\frac{1}{4} \times\left(a_{i}-d+b_{i}-c+c_{i}-b+d_{i}-a\right) \times\left(\mu_{\bar{A} ! R}-v_{\bar{A} ! R}\right) . \alpha, \beta$ are risk attitude coefficientsshowing the concave-convex degree of region value power function of the gains and the losses, 
$0 \leq \alpha, \beta \leq 1, \theta$ is loss aversion coefficient which represents that the region value function is steeper for the losses than the gains.

Definition 7 [12]. Suppose that $\mu$ is the fuzzy measure in the universe of discourse $X=\left\{x_{1}, x_{2}, \ldots, x_{n}\right\}$ and $v\left(\Delta A_{i}\right)$ is a set of intuitionistic trapezoidal fuzzy prospect value function of $\AA_{i}=\left(\left[a_{i}, b_{i}, c_{i}, d_{i}\right] ; \mu_{i}, v_{i}\right)(i=1,2, \ldots, n)$. Then the discrete Choquet integral of $v\left(\Delta \AA_{i}\right)$ on fuzzy measure $\mu$ is defined as follows:

$\int v\left(\Delta \AA_{i}\right) d u=\operatorname{ITFCI}\left(v\left(\Delta \AA_{1}\right), v\left(\Delta \AA_{2}\right), \ldots, v\left(\Delta \AA_{n}\right)\right)$

$=v\left(\Delta \bar{A}_{\tau(1)}\right)\left[\mu\left(B_{\tau(1)}\right)-\mu\left(B_{\tau(2)}\right)\right] \oplus \ldots \oplus v\left(\Delta \AA_{\tau(n)}\right)\left[\mu\left(B_{\tau(n)}\right)-\mu\left(B_{\tau(n+1)}\right)\right]$

$=\sum_{i=1}^{n} v\left(\Delta \AA_{i}\right)\left[\mu\left(B_{\tau(i)}\right)-\mu\left(B_{\tau(i+1)}\right)\right]$

Where $v\left(\Delta \AA_{\tau(1)}\right), v\left(\Delta \AA_{\tau(2)}\right), \ldots, v\left(\Delta \AA_{\tau(n)}\right)$ is the permutation of $v\left(\Delta \AA_{1}\right), v\left(\Delta \AA_{2}\right), \ldots, v\left(\Delta \AA_{n}\right)$, for any $i$, $v\left(\Delta \AA_{\tau(i)}\right) \leq v\left(\Delta \AA_{\tau(i+1)}\right) ; B_{\tau(i)}=\left(x_{\tau(i)}, x_{\tau(i+1)}, \ldots, x_{\tau(n)}\right), B_{\tau(n+1)}=0$.

\section{Decision-Making Method And Step}

$D M=\left\{D M_{1}, D M_{2}, \ldots, D M_{k}, \ldots, D M_{h}\right\}, k=1,2, \ldots, h$ are decision makers to select the best wind investment alternative from $m$ alternatives $A_{i}(i=1,2, \ldots, m)$ assessed on $n$ attributes $C_{j}(j=1,2, \ldots, n)$.

Step1. Obtain intuitionistic trapezoidal fuzzy decision matrixes given by decision makers, which can be denoted by:

$X^{1}=\left[\tilde{x}_{i j}^{1}\right]_{m \times n}, X^{2}=\left[\tilde{x}_{i j}^{2}\right]_{m \times n}, \ldots, X^{h}=\left[\tilde{x}_{i j}^{h}\right]_{m \times n}$

where $\tilde{x}_{i j}^{k}=\left(\left[a_{i j}^{k}, b_{i j}^{k}, c_{i j}^{k}, d_{i j}^{k}\right] ; \mu_{i j}^{k}, v_{i j}^{k}\right)$ is the performance of alternative $A_{i}$ under attribute $C_{j}$ provided by $D M_{k}$ with ITFN.

Step2. According to the prospect theory, decision makers give their different reference points $R_{j}^{k}=\left(\left[a_{j}^{k}, b_{j}^{k}, c_{j}^{k}, d_{j}^{k}\right] ; \mu_{j}^{k}, v_{j}^{k}\right)$ based on different attributes $C_{j}(i=1,2, \ldots, n)$. According to Definition 5, the deviation degree between $\tilde{x}_{i j}^{k}$ and $R_{j}^{k}$ is :

$$
\tilde{\Delta x_{i j}^{k}}=\tilde{x}_{i j}^{k} ! R_{j}^{k}=\left(\left[a_{i j}^{k}-d_{j}^{k}, b_{i j}^{k}-c_{j}^{k}, c_{i j}^{k}-b_{j}^{k}, d_{i j}^{k}-a_{j}^{k}\right] ; \mu_{\tilde{x}_{i j}^{k} ! R_{j}^{k}}, v_{\tilde{x}_{x_{j}} ! R_{j}^{k}}\right)
$$


Step3. According to Definition 7, intuitionistic trapezoidal fuzzy decision matrixes $X^{1}=\left[\tilde{x}_{i j}^{1}\right]_{m \times n}$, $X^{2}=\left[\tilde{x}_{i j}^{2}\right]_{m \times n}, \ldots, X^{h}=\left[\tilde{x}_{i j}^{h}\right]_{m \times n}$ are transformed to intuitionistic trapezoidal fuzzy decision prospect matrixes $P X^{1}=\left[v\left(\Delta \tilde{x}_{i j}^{1}\right)\right]_{m \times n}, P X^{2}=\left[v\left(\Delta \tilde{x}_{i j}^{2}\right)\right]_{m \times n}, \ldots, P X^{h}=\left[v\left(\Delta \tilde{x}_{i j}^{h}\right)\right]_{m \times n}$ by the deviation degree.

Where

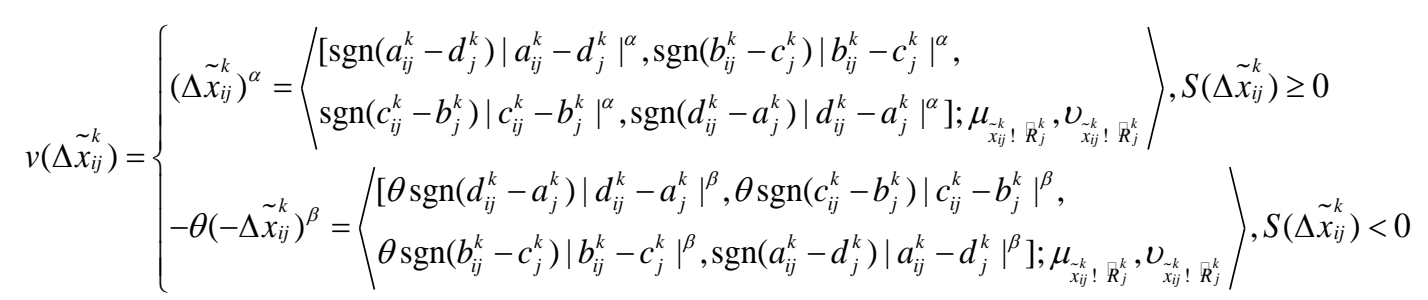

Step4. By means of history sample information, the fuzzy measure is obtained. And then the intuitionistic trapezoidal fuzzy decision prospect matrixes are aggregated by the intuitionistic trapezoidal fuzzy Choquet integral prospect operator. The nuts and bolts are:

$$
\begin{aligned}
& \int v\left(\Delta \tilde{x}_{i j}^{k}\right) d u=\operatorname{CITFCI}\left(v\left(\Delta \tilde{x}_{i j}^{1}\right), v\left(\Delta \tilde{x}_{i j}^{2}\right), \ldots, v\left(\Delta \tilde{x}_{i j}^{h}\right)\right) \\
& =v\left(\Delta \tilde{x}_{i j}^{\tau(1)}\right)\left[\mu\left(D M_{\tau(1)}\right)-\mu\left(D M_{\tau(2)}\right)\right] \oplus \ldots \oplus v\left(\Delta \tilde{x}_{i j}^{\tau(h)}\right)\left[\mu\left(D M_{\tau(h)}\right)-\mu\left(D M_{\tau(h+1)}\right)\right] \\
& =\sum_{k=1}^{h} v\left(\Delta \tilde{x}_{i j}^{\tau(k)}\right)\left[\mu\left(D M_{\tau(k)}\right)-\mu\left(D M_{\tau(k+1)}\right)\right]
\end{aligned}
$$

Where $v\left(\tilde{x}_{i j}^{\tau(1)}\right), v\left(\tilde{x}_{i j}^{\tau(2)}\right), \ldots, v\left(\Delta \tilde{x}_{i j}^{\tau(h)}\right)$ is the permutation of $v\left(\tilde{x}_{i j}^{1}\right), v\left(\Delta \tilde{x}_{i j}^{2}\right), \ldots, v\left(\Delta \tilde{x}_{i j}^{h}\right)$. And then, the comprehensive prospect decision matrix $C P X=\left(\left[e p x_{i j}\right)_{m \times n}\right.$ is obtained.

$$
\begin{aligned}
& C P X=\left(\sum p x_{i j}\right)_{m \times n}=\left[\operatorname{CITFNCI}\left(v\left(\Delta \tilde{x}_{i j}^{1}\right), v\left(\Delta \tilde{x}_{i j}^{2}\right), \ldots, v\left(\Delta \tilde{x}_{i j}^{h}\right)\right)\right]_{m \times n} \\
& =\left\{\begin{array}{l}
\left(p a_{i j}, p b_{i j}, p c_{i j}, p d_{i j} ; p \mu_{i j}, p v_{i j}\right), S\left(v\left(\Delta \tilde{x}_{i j}^{k}\right)\right) \geq 0 \\
\left(p a_{i j}^{\theta}, p b_{i j}^{\theta}, p c_{i j}^{\theta}, p d_{i j}^{\theta} ; p \mu_{i j}^{\theta}, p v_{i j}^{\theta}\right), S\left(v\left(\Delta \tilde{x}_{i j}^{k}\right)\right)<0
\end{array}\right.
\end{aligned}
$$

Where

$$
p a_{i j}=\sum_{k=1}^{h} \operatorname{sgn}\left(a_{i j}^{\tau(k)}-d_{j}^{\tau(k)}\right)\left|a_{i j}^{\tau(k)}-d_{j}^{\tau(k)}\right|^{\alpha} \Delta \mu_{\tau(k)}, p b_{i j}=\sum_{k=1}^{h} \operatorname{sgn}\left(b_{i j}^{\tau \tau(k)}-c_{j}^{\tau(k)}\right)\left|b_{i j}^{\tau(k)}-c_{j}^{\tau(k)}\right|^{\alpha} \Delta \mu_{\tau(k)},
$$


International Journal of Fuzzy Logic Systems (IJFLS) Vol.7, No.2, October 2017

$$
\begin{aligned}
& p c_{i j}=\sum_{k=1}^{h} \operatorname{sgn}\left(c_{i j}^{\tau(k)}-b_{j}^{\tau(k)}\right)\left|c_{i j}^{\tau(k)}-b_{j}^{\tau(k)}\right|^{\alpha} \Delta \mu_{\tau(k)}, p d_{i j}=\sum_{k=1}^{h} \operatorname{sgn}\left(d_{i j}^{\tau(k)}-a_{j}^{\tau(k)}\right)\left|d_{i j}^{\tau(k)}-a_{j}^{\tau(k)}\right|^{\alpha} \Delta \mu_{\tau(k)}, \\
& p \mu_{i j}=\frac{\sum_{k=1}^{h}\left[\left\|\Delta \tilde{x}_{i j}^{\tau(k)}\right\|\right]^{\alpha}\left(\mu_{x R}\right)_{\tau(k)}}{\sum_{k=1}^{h}\left[\left\|\Delta \tilde{x}_{i j}^{\tau(k)}\right\|\right]^{\alpha}}, p v_{i j}=\frac{\sum_{k=1}^{h}\left[\left\|\Delta \tilde{x}_{i j}^{\tau(k)}\right\|\right]^{\alpha}\left(v_{x R}\right)_{\tau(k)}}{\sum_{k=1}^{h}\left[\left\|\Delta \tilde{x}_{i j}^{\tau(k)}\right\|\right]^{\alpha}}, \\
& p a_{i j}^{\theta}=\sum_{k=1}^{h} \theta \operatorname{sgn}\left(d_{i j}^{\tau(k)}-a_{j}^{\tau(k)}\right)\left|d_{i j}^{\tau(k)}-a_{j}^{\tau(k)}\right|^{\beta} \Delta \mu_{\tau(k)}, p b_{i j}^{\theta}=\sum_{k=1}^{h} \theta \operatorname{sgn}\left(c_{i j}^{\tau(k)}-b_{j}^{\tau(k)}\right)\left|c_{i j}^{\tau(k)}-b_{j}^{\tau(k)}\right|^{\beta} \Delta \mu_{\tau(k)} \\
& p c_{i j}^{\theta}=\sum_{k=1}^{h} \theta \operatorname{sgn}\left(b_{i j}^{\tau(k)}-c_{j}^{\tau(k)}\right)\left|b_{i j}^{\tau(k)}-c_{j}^{\tau(k)}\right|^{\beta} \Delta \mu_{\tau(k)} \\
& p d_{i j}^{\theta}=\sum_{k=1}^{h} \theta \operatorname{sgn}\left(a_{i j}^{\tau(k)}-d_{j}^{\tau(k)}\right)\left|a_{i j}^{\tau(k)}-d_{j}^{\tau(k)}\right|^{\beta} \Delta \mu_{\tau(k)} \\
& p \mu_{i j}^{\theta}=\frac{\sum_{k=1}^{h}\left[-\theta\left\|\Delta \tilde{x}_{i j}^{\tau(k)}\right\|\right]^{\beta}\left(\mu_{x R}\right)_{\tau(k)}}{\sum_{k=1}^{h}\left[-\theta\left\|\Delta \tilde{x}_{i j}^{\tau(k)}\right\|\right]^{\beta}}, \quad p v_{i j}^{\theta}=\frac{\sum_{k=1}^{h}\left[-\theta\left\|\Delta \tilde{x}_{i j}^{\tau(k)}\right\|\right]^{\beta}\left(\mu_{x R}\right)_{\tau(k)}}{\sum_{k=1}^{h}\left[-\theta\left\|\Delta \tilde{x}_{i j}^{\tau(k)}\right\|\right]^{\beta}} . \\
& \Delta \mu_{\tau(k)}=\mu\left(H_{\tau(k)}\right)-\mu\left(H_{\tau(k+1)}\right), \quad i=1,2, \ldots, m, j=1,2, \ldots, n .
\end{aligned}
$$

Step5. Normalize the comprehensive prospect decision matrix.

$$
\begin{aligned}
& N C P X=\left(n c p x_{i j}\right)_{m \times n}=\left[\operatorname{NCITFNCI}\left(v\left(\Delta \tilde{x}_{i j}^{1}\right), v\left(\Delta \tilde{x}_{i j}^{2}\right), \ldots, v\left(\Delta \tilde{x}_{i j}^{h}\right)\right)\right]_{m \times n} \\
& =\left\{\begin{array}{l}
\left(\frac{p a_{i j}}{D_{j}}, \frac{p b_{i j}}{D_{j}}, \frac{p c_{i j}}{D_{j}}, \frac{p d_{i j}}{D_{j}} ; p \mu_{i j}, p v_{i j}\right), S\left(v\left(\Delta \tilde{x}_{i j}^{k}\right)\right) \geq 0 \\
\left(\frac{p a_{i j}^{\theta}}{D_{j}^{\theta}}, \frac{p b_{i j}^{\theta}}{D_{j}^{\theta}}, \frac{p c_{i j}^{\theta}}{D_{j}^{\theta}}, \frac{p d_{i j}^{\theta}}{D_{j}^{\theta}} ; p \mu_{i j}^{\theta}, p v_{i j}^{\theta}\right), S\left(v\left(\Delta \tilde{x}_{i j}^{k}\right)\right)<0
\end{array}\right. \\
& =\left(p a_{i j}^{*}, p b_{i j}^{*}, p c_{i j}^{*}, p d_{i j}^{*} ; p \mu_{i j}^{*}, p v_{i j}^{*}\right)
\end{aligned}
$$

Where $D_{j}=\left(\sum_{i=1}^{m}\left[\left(p a_{i j}\right)^{2}+\left(p b_{i j}\right)^{2}+\left(p c_{i j}\right)^{2}+\left(p d_{i j}\right)^{2}\right]\right)^{\frac{1}{2}}$,

$$
D_{j}^{\theta}=\left(\sum_{i=1}^{m}\left[\left(p a_{i j}^{\theta}\right)^{2}+\left(p b_{i j}^{\theta}\right)^{2}+\left(p c_{i j}^{\theta}\right)^{2}+\left(p d_{i j}^{\theta}\right)^{2}\right]\right)^{\frac{1}{2}}, i=1,2, \ldots, m, j=1,2, \ldots, n
$$

Step6. Calculate the distance between the normalized comprehensive prospect decision information $n c p x_{i j}$ and the normalized ideal reference vector $R^{*}=([1,1,1,1] ; 1,0)$. 


$$
\begin{array}{r}
d_{i j}=\frac{1}{8}\left(\left|\left(1+p \mu_{i j}^{*}-p v_{i j}^{*}\right) p a_{i j}^{*}-2\right|+\left|\left(1+p \mu_{i j}^{*}-p v_{i j}^{*}\right) p b_{i j}^{*}-2\right|+\right. \\
\left.\left|\left(1+p \mu_{i j}^{*}-p v_{i j}^{*}\right) p c_{i j}^{*}-2\right|+\left|\left(1+p \mu_{i j}^{*}-p v_{i j}^{*}\right) p d_{i j}^{*}-2\right|\right)
\end{array}
$$

Step7. Calculate the grey correlation coefficient $r_{i j}$ :

$$
r_{i j}=\frac{d_{\min }+0.5 * d_{\max }}{d_{i j}+0.5 * d_{\max }}
$$

Where $d_{\text {max }}=\max \left\{d_{i j}\right\}, d_{\text {min }}=\min \left\{d_{i j}\right\}$.

Step8. Construct the optimization model to obtain the best projection vector $\omega_{j}$.

$$
\begin{aligned}
& \max \sum_{j=1}^{n} \omega_{j} r_{i j} \\
& \text { s.t. } \sum_{j=1}^{n} \omega_{j}^{2}=1, \omega_{j}^{2} \geq 0
\end{aligned}
$$

Step9. Calculate the grey correlation projection value $z_{i}$.

Draw the experience from[17], the cosine vector of the projection angle between the alternative $A_{i}$ and the normalized ideal reference vector is:

$$
\chi_{i}=\cos \alpha=\frac{\sum_{j=1}^{n} \omega_{j} r_{i j} \omega_{j}}{\sqrt{\sum_{j=1}^{n}\left[\omega_{j} r_{i j}\right]^{2}} \sqrt{\sum_{j=1}^{n} \omega_{j}^{2}}}
$$

Obviously, $0 \leq d_{i} \leq 1$, the bigger the better.

The grey correlation projection value $z_{i}$ between the alternative $A_{i}$ and the normalized ideal reference vector is:

$$
z_{i}=M\left(A_{i}\right) \square \chi_{i}=\frac{\sum_{j=1}^{n} \omega_{j}^{2} r_{i j}}{\sqrt{\sum_{j=1}^{n} \omega_{j}^{2}}}=\sum_{j=1}^{n} \bar{\omega}_{j} r_{i j}
$$

Where $M\left(A_{i}\right)=\sum_{j=1}^{n}\left[\omega_{j} r_{i j}\right]^{2}$ is the moduli of $A_{i}$. And $\bar{\omega}_{j}$ is the grey correlation projection vector, and it can be calculated by: 
International Journal of Fuzzy Logic Systems (IJFLS) Vol.7, No.2, October 2017

$$
\bar{\omega}_{j}=\frac{\omega_{j}^{2}}{\sqrt{\sum_{j=1}^{n} \omega_{j}^{2}}}
$$

According to the grey correlation projection value $z_{i}$, we can rank the alternatives.

\section{A Real Example}

Jilin province plan to invest a wind energy project in Baicheng. There are four alternative projects $\left\{A_{1}, A_{2}, A_{3}, A_{4}\right\}$. Four attributes are selected, including technology capability $C_{1}$, economy benefit $C_{2}$, social influence $C_{3}$ and environment management $C_{4}$, which are all benefit oriented attributes. The power supply company of Jilin invites three experts to form a group of decision makers.

Step 1. Three experts are invited to evaluate the four alternatives under the attributes. They give their evaluation information with ITFNs in table 1-3.

Table1 The initial decision information given by $D M_{1}$

\begin{tabular}{|l|l|l|l|l|}
\hline & $C_{1}$ & $C_{2}$ & $C_{3}$ & $C_{4}$ \\
\hline$A_{1}$ & $([2,3,5,7] ; 0.6,0.3)$ & $([3,5,7,8] ; 0.7,0.2)$ & $([2,5,6,7] ; 0.7,0.2)$ & $([3,4,5,8] ; 0.5,0.2)$ \\
\hline$A_{2}$ & $([4,6,7,9] ; 0.4,0.2)$ & $([3,4,5,9] ; 0.5,0.1)$ & $([3,4,5,6] ; 0.6,0.1)$ & $([2,4,5,7] ; 0.7,0.1)$ \\
\hline$A_{3}$ & $([1,3,4,5] ; 0.7,0.3)$ & $([2,5,7,8] ; 0.8,0.1)$ & $([3,4,6,8] ; 0.5,0.2)$ & $([5,7,8,9] ; 0.9,0.1)$ \\
\hline$A_{4}$ & $([3,4,7,8] ; 0.5,0.2)$ & $([1,3,6,8] ; 0.7,0.3)$ & $([3,4,5,6] ; 0.6,0.2)$ & $([2,3,6,7] ; 0.8,0.1)$ \\
\hline
\end{tabular}

Table 2 The initial decision information given by $D M_{2}$

\begin{tabular}{|l|l|l|l|l|}
\hline & $C_{1}$ & $C_{2}$ & $C_{3}$ & $C_{4}$ \\
\hline$A_{1}$ & $([2,4,7,9] ; 0.7,0.2)$ & $([2,3,5,7] ; 0.6,0.2)$ & $([2,3,5,8] ; 0.7,0.1)$ & $([2,3,6,8] ; 0.6,0.2)$ \\
\hline$A_{2}$ & $([4,5,8,9] ; 0.5,0.2)$ & $([3,5,7,8] ; 0.7,0.1)$ & $([3,6,7,9] ; 0.8,0.1)$ & $([2,5,8,9] ; 0.8,0.1)$ \\
\hline$A_{3}$ & $([2,4,6,8] ; 0.6,0.1)$ & $([2,3,4,6] ; 0.5,0.2)$ & $([1,2,4,6] ; 0.6,0.3)$ & $([3,4,6,7] ; 0.7,0.2)$ \\
\hline$A_{4}$ & $([2,3,4,6] ; 0.6,0.2)$ & $([2,5,6,9] ; 0.8,0.1)$ & $([3,5,6,7] ; 0.7,0.2)$ & $([2,3,5,8] ; 0.6,0.1)$ \\
\hline
\end{tabular}

Table 3 The initial decision information given by $D M_{3}$

\begin{tabular}{|l|l|l|l|l|}
\hline & $C_{1}$ & $C_{2}$ & $C_{3}$ & $C_{4}$ \\
\hline$A_{1}$ & $([4,5,7,8] ; 0.7,0.1)$ & $([2,4,5,8] ; 0.7,0.2)$ & $([2,5,6,9] ; 0.8,0.1)$ & $([4,5,6,9] ; 0.7,0.1)$ \\
\hline$A_{2}$ & $([1,3,6,8] ; 0.8,0.2)$ & $([2,3,6,7] ; 0.6,0.1)$ & $([2,3,5,8] ; 0.7,0.1)$ & $([1,2,3,4] ; 0.6,0.1)$ \\
\hline$A_{3}$ & $([4,5,7,8] ; 0.6,0.2)$ & $([1,4,5,7] ; 0.5,0.2)$ & $([1,2,5,7] ; 0.6,0.2)$ & $([3,6,7,10] ; 0.7,0.1)$ \\
\hline$A_{4}$ & $([2,3,5,6] ; 0.7,0.1)$ & $([2,3,5,6] ; 0.7,0.2)$ & $([4,5,7,8] ; 0.5,0.1)$ & $([4,7,8,9] ; 0.6,0.3)$ \\
\hline
\end{tabular}


Step 2. Table 4 shows reference points given by experts. The reference points are the ideal values by decision makers. For example, $D M_{1}$ thinks that the value of the ideal project under attribute $C_{1}$ is $([1.68,3.53,4.95,8.04] ; 0.75,0.15)$

Table 4 The reference points

\begin{tabular}{|l|l|l|}
\hline & $C_{1}$ & $C_{2}$ \\
\hline$D M_{1}$ & $([1.68,3.53,4.95,8.04] ; 0.75,0.15)$ & $([1.72,3.78,6.25,7.64] ; 0.65,0.30)$ \\
\hline$D M_{2}$ & $([2.31,4.05,6.27,7.78] ; 0.65,0.25)$ & $([2.23,4.22,5.76,7.87] ; 0.75,0.15)$ \\
\hline$D M_{3}$ & $([1.54,3.28,5.35,6.76] ; 0.65,0.10)$ & $([1.68,3.42,4.78,6.39] ; 0.65,0.20)$ \\
\hline & $C_{3}$ & $C_{3}$ \\
\hline$D M_{1}$ & $([2.24,3.39,5.47,7.14] ; 0.75,0.15)$ & $([2.42,3.63,5.25,6.57] ; 0.65,0.20)$ \\
\hline$D M_{2}$ & $([1.94,3.23,4.83,6.45] ; 0.65,0.20)$ & $([1.46,2.87,4.63,6.49] ; 0.75,0.15)$ \\
\hline$D M_{3}$ & $([2.12,4.62,6.14,7.35] ; 0.75,0.15)$ & $([1.64,3.52,5.35,6.51] ; 0.75,0.15)$ \\
\hline
\end{tabular}

Step3. According to [12], $\alpha=0.89, \beta=0.92, \sigma=2.25$. After calculating the prospect value function by (8), we transform the intuitionistic trapezoidal fuzzy decision matrixes to intuitionistic trapezoidal fuzzy prospect matrixes. Due to space limitation, the process is omitted.

Step4. The fuzzy measures are obtained according to history sample information. $\mu(\varnothing)=0$, $\mu\left(\left\{\mathrm{DM}_{1}\right\}\right)=0.3 \quad, \quad \mu\left(\left\{\mathrm{DM}_{2}\right\}\right)=0.4, \mu\left(\left\{\mathrm{DM}_{1}\right\}\right)=0.5, \mu\left(\left\{\mathrm{DM}_{1}, \mathrm{DM}_{2}\right\}\right)=0.6, \mu\left(\left\{\mathrm{DM}_{1}, \mathrm{DM}_{3}\right\}\right)=0.6$, $\mu\left(\left\{\mathrm{DM}_{3}, \mathrm{DM}_{2}\right\}\right)=0.7, \mu\left(\left\{\mathrm{DM}_{1}, \mathrm{DM}_{2}, \mathrm{DM}_{3}\right\}\right)=1$. The comprehensive prospect decision matrix is obtained by (9) shown in table5.

Table5 The comprehensive prospect decision matrix

\begin{tabular}{|l|l|l|}
\hline & $C_{1}$ & $C_{2}$ \\
\hline$A_{1}$ & $([-5.75,-3.36,0.78,3.48] ; 0.68,0.21)$ & $([-5.18,-2.54,1.83,4.53] ; 0.72,0.18)$ \\
\hline$A_{2}$ & $([-4.83,-1.89,1.25,5.24] ; 0.68,0.19)$ & $([-2.87,-1.23,2.93,5.21] ; 0.68,0.15)$ \\
\hline$A_{3}$ & $([-3.58,-1.25,2.32,5.74] ; 0.65,0.17)$ & $([-2.98,-1.15,2.57,5.63] ; 0.71,0.16)$ \\
\hline$A_{4}$ & $([-4.27,-2.68,1.24,4.83] ; 0.68,0.21)$ & $([-5.32,-2.46,2.34,6.57] ; 0.67,0.17)$ \\
\hline & $C_{3}$ & $C_{3}$ \\
\hline$A_{1}$ & $([-6.54,-4.18,2.58,5.79] ; 0.72,0.18)$ & $([-6.53,-3.26,3.06,7.27] ; 0.69,0.17)$ \\
\hline$A_{2}$ & $([-4.67,-2.25,-1.04,3.76] ; 0.69,0.14)$ & $([-5.85,-4.52,1.78,5.24] ; 0.68,0.19)$ \\
\hline$A_{3}$ & $([-5.54,-1.72,1.57,4.82] ; 0.69,0.20)$ & $([-3.79,-0.34,3.27,5.84] ; 0.74,0.18)$ \\
\hline$A_{4}$ & $([-3.21,-1.86,1.86,5.27] ; 0.70,0.16)$ & $([-7.43,-1.96,2.54,6.32] ; 0.67,0.22)$ \\
\hline
\end{tabular}

Step 5. The comprehensive prospect decision matrix is normalized by (11). The process is omitted Step6-7. By (12)-(13), the distance and grey correlation coefficients are calculated shown in Table6. 
Table6. The distance and grey correlation coefficients

\begin{tabular}{|l|l|l|l|l|l|l|l|l|}
\hline & \multicolumn{9}{|l|}{$d_{\bar{j}}$} & \multicolumn{2}{l|}{$r_{\text {ij }}$} \\
\hline & $C_{1}$ & $C_{2}$ & $C_{3}$ & $C_{4}$ & $C_{1}$ & $C_{2}$ & $C_{3}$ & $C_{4}$ \\
\hline$A_{1}$ & 1.170 & 0.753 & 1.047 & 1.227 & 0.847 & 0.967 & 0.887 & 0.876 \\
\hline$A_{2}$ & 1.034 & 0.886 & 1.123 & 1.087 & 0.913 & 0.939 & 0.824 & 0.934 \\
\hline$A_{3}$ & 0.887 & 0.857 & 1.032 & 0.786 & 0.894 & 0.897 & 0.914 & 1.000 \\
\hline$A_{4}$ & 1.107 & 1.045 & 0.918 & 1.028 & 0.906 & 0.894 & 0.907 & 0.927 \\
\hline
\end{tabular}

Step8. Set the parameters of the optimization model, $m=4, n=4$, and the cluster is divided into two categories. Then the best projection vector is $\omega_{j}=(0.6347,0.36142,0.4722,0.6157)$.

Step9. By (17), the grey correlation projection vector $\bar{\omega}_{j}=(0.2687,0.1045,0.3658,0.1610)$ is obtained. The grey correlation projection values of alternatives are computed $: z_{1}=0.8837$, $z_{2}=0.8956, z_{3}=0.9037, z_{4}=0.9107$. The ranking result is: $A_{4} \succ A_{3} \succ A_{2} \succ A_{1}$, so the best appropriate alternative is $A_{4}$.

\section{Conclusion}

This paper presents a MADM model with ITFN based on Choquet integral and prospect theory for selecting the best wind energy investment project in Jilin province. Intuitionistic trapezoidal fuzzy number is good at describing the uncertainty information in MADM problem. For the problem of interdependency in attributes, we use fuzzy Choquet integral to improve ITFNs. In consideration of decision makers' bounded rationality, the intuitionistic trapezoidal fuzzy Choquet integral prospect (ITFCIP) operator is defined to aggregate the ITFNs. In the case study, a wind energy investment project selection problem is calculated by the proposed method.

Another important point for this study is the introduction of Choquet integral and prospect theory to ITFN, which an important contribution to MCDM literature. One of the practical implications of this study is that the presented methodology can be used for multi-criteria evaluation problems rather wind energy decision by modifying the evaluation criteria.

\section{ACKNOWLEDGEMENT:}

The authors would like to acknowledge the supports from National Natural Science Foundation of China (No. 71271084, No. 71671065) and the technology project of State Grid (5204BB1600CN).

\section{REFERENCES:}

[1] Sebitosi, A.B., Energy efficiency, security of supply and the environment in South Africa: Moving beyond the strategy documents. Energy, 2008. 33(11): p. 1591-1596. 
[2] Pacala, S. and R. Socolow, Stabilization Wedges: Solving the Climate Problem for the Next 50 Years with Current Technologies. Science, 2004. 305(5686): p. 968-972.

[3] Barry, M.L., H. Steyn and A. Brent, Selection of renewable energy technologies for Africa: Eight case studies in Rwanda, Tanzania and Malawi. Renewable Energy, 2011. 36(11): p. 2845-2852.

[4] Aydin, N.Y., E. Kentel and H.S. Duzgun, GIS-based site selection methodology for hybrid renewable energy systems: A case study from western Turkey. Energy Conversion \& Management, 2013. 70(70): p. 90-106.

[5] Baños, R., et al., Optimization methods applied to renewable and sustainable energy: A review. Renewable \& Sustainable Energy Reviews, 2011. 15(4): p. 1753-1766.

[6] Sagbansua, L. and F. Balo, Decision making model development in increasing wind farm energy efficiency. Renewable Energy, 2017. 109: p. 354-362.

[7] Łaska, G., Wind Energy and Multi-criteria Analysis in Making Decisions on the Location of Wind Farms. Procedia Engineering, 2017. 182: p. 418-424.

[8] Villacreses, G., et al., Wind farms suitability location using geographical information system (GIS), based on multi-criteria decision making (MCDM) methods: The case of continental Ecuador. Renewable Energy, 2017. 109: p. 275-286.

[9] Elzarka, H.M., H. Yan and D. Chakraborty, A vague set fuzzy multi-attribute group decision-making model for selecting onsite renewable energy technologies for institutional owners of constructed facilities. Sustainable Cities \& Society, 2017. 35: p. 430-439.

[10] Yuan, J. and C. Li, A New Method for Multi-Attribute Decision Making with Intuitionistic Trapezoidal Fuzzy Random Variable. International Journal of Fuzzy Systems, 2017. 19(1): p. 15-26.

[11] Chen, Z.S. and Y.L. Li, Approach for group MULTIMOORA decision making based upon prospect intuitionistic trapezoidal fuzzy number Choquet integral operator. Control \& Decision, 2014. 29(6): p. 1053-1063.

[12] Yuan J. H, L.C.B., Intuitionistic Trapezoidal Fuzzy Group Decision-Making Based on Prospect Choquet Integral Operator and Grey Projection Pursuit Dynamic Cluster. Mathematical Problems in Engineering, 2017. 2017(12): p. 1-13. 